\title{
L'importanza crescente delle Nuove Vie della Seta nel dibattito geografico e nelle altre discipline
}

Dino Gavinelli

Università degli Studi di Milano

DOI: http://dx.doi.org/10.7358/gn-2018-001-gav1

I discorsi istituzionali esplicitati in diversi contesti negli ultimi anni e messi in essere intorno alle cosiddette Nuove Vie della Seta (più conosciute in inglese con i termini Belt and Road Initiative - BRI e anche One Belt One Road - OBOR) occupano una posizione di crescente importanza nelle narrazioni contemporanee. Da più parti si sottolineano infatti le ricadute positive per i paesi asiatici, europei, africani e del Pacifico meridionale coinvolti nei numerosi progetti già conclusi, avviati o pianificati. Il rafforzamento degli scambi politici, economici, commerciali e culturali, il potenziamento delle reti di collegamento terrestri e marittime, la realizzazione di nuove infrastrutture per i trasporti e le inevitabili trasformazioni territoriali previste da questo imponente progetto di portata mondiale e volto a percorrere la difficile strada di un modello cosiddetto win-win, hanno spinto la Direzione e il Comitato scientifico di Geography Notebooks, Quaderni di Geografia, Cabiers de Géographie, Cuadernos de Geografía, a dedicare il primo numero della rivista a questo tema. Le Nuove Vie della Seta stanno infatti ridisegnando in profondità gli equilibri del mondo contemporaneo in più direzioni. Si deve tenere poi in conto il fatto che la costruzione di un vasto sistema di trasporti e comunicazioni (strade, ferrovie, porti, aeroporti, oleodotti e gasdotti) e la realizzazione di numerose infrastrutture (con la messa in opera di una rete di comunicazioni telematiche ed informatiche per migliorare la connessione regionale) non solo potenzierà l'integrazione politica ed economica euroasiatica, ridiscuterà i termini della partnership di molti stati con l'Africa, ridisegnerà gli equilibri geopolitici mondiali, 
rafforzerà il ruolo di riferimento della Cina in quanto attore con il più grande peso demografico ed economico all'interno del progetto, ma più in generale favorirà da un lato una cooperazione interstatale e un discorso multilaterale ma dall'altro anche resistenze da parte degli esclusi o degli oppositori. Tutto questo alimenterà anche speranze che andranno più o meno deluse o, al contrario, soddisfatte.

Per meglio affrontare alcune tematiche e delineare la portata del progetto in corso di realizzazione si è pensato di dare spazio, secondo lo spirito della rivista, a più approcci disciplinari. Ecco perché nel presente numero a fianco della lettura geografica e geostorica delle Vecchie e delle Nuove Vie della Seta si sono affiancate, senza pretesa di esaustività su un tema così ampio e in divenire, quelle di esperti di comunicazione, linguaggi dei media, manifestazioni culturali, economia e relazioni internazionali. Alcuni di essi, ad esempio, evidenziano come l'iniziativa abbia una grande potenzialità perché promuoverà la cooperazione monetaria tra gli stati coinvolti (oltre una sessantina) e a dare allo yuan renminbi, la divisa cinese, lo statuto innovativo di moneta di scambio e di riferimento nelle transazioni economiche e finanziarie alla scala asiatica e persino mondiale. Altri contributi invece sottolineano come sarà inevitabile pensare all'iniziativa non solo secondo una semplice visione storica, economica, logistica, dei trasporti e delle comunicazioni. Il coinvolgimento crescente delle classi politiche e di governo degli stati coinvolti, degli investitori, delle élite culturali e delle masse ne sono una prima testimonianza. Anche le ricadute in politica estera e il ridisegno dei poteri militari sono evidenti in caso di successo del progetto: i rapporti diplomatici e gli scambi culturali tra gli stati coinvolti ne risulterebbero stravolti; l'influenza cinese negli equilibri geopolitici crescerebbe notevolmente in tutte le direzioni; e l'immagine della Cina acquisirebbe nuovo slancio in vista del centenario della fondazione della Repubblica Popolare del 2049.

Dal punto di vista della politica cinese, l'iniziativa delle Nuove Vie della Seta spinge per un grande discorso nazionale che coinvolge le grandi masse popolari e che colloca saldamente Pechino nella sua dimensione geopolitica ed economica tradizionale di paese 'al centro dell'Asia'. Interessante anche osservare come la dimensione terrestre del progetto induca i responsabili cinesi a promuovere importanti politiche di riequilibrio socio-territoriali, economiche e infrastrutturali tra le province orientali densamente popolate e ricche e quelle occidentali che appaiono decisamente più spopolate, povere e sotto dotate dal punto di vista infrastrutturale e logistico. La dimensione marittima del progetto vede invece la Cina impegnata a consolidare la sua posizione sui mari asiatici e a delimitare un perimetro di sicurezza per 
i suoi approvvigionamenti, con la costruzione di una rete di 'presidi' portuali lungo la vitale linea marittima che unisce i porti cinesi con l'Oceano Indiano, il Golfo Persico, il Mar Rosso e il Mediterraneo. Infine anche gli aspetti ambientali, energetici e dello sfruttamento delle risorse non sono da sottovalutare sul medio-lungo periodo per la Cina, perché l'apertura di nuove vie terrestri e marittime potrebbe sostenere anche una maggiore diversificazione delle fonti di approvvigionamento energetico e di materie prime, e quindi il superamento della dipendenza da una sola regione o da un solo fornitore.

A conclusione del primo numero è parso utile affiancare ai contributi scientifici forniti dai diversi autori anche una dimensione didattica e educativa. Appare infatti evidente come la Belt and Road Initiative si presti a numerose riflessioni e applicazioni didattiche, essendo una realtà che difficilmente gli studenti, futuri cittadini attivi dei prossimi decenni, non potranno non conoscere. Le diverse discipline scolastiche (storia, economia, geografia, diritto, solo per citare quelle più stimolate dalle opportunità e dalle sfide offerte dalla BRI) possono dare il loro prezioso contributo, in termini di metodi, obiettivi e strumenti didattici, per avvicinare allo studio e alla conoscenza di quello che potrebbe diventare il più importante esperimento socio-economico e politico-sociale del Terzo Millennio o rivelarsi, al contrario, una delle più grandi utopie di integrazione regionale che ha provato inutilmente a sostituire l'economia e la collaborazione allo scontro e alle guerre tra gli stati. 
